\title{
A Simple Extraction Method of Young's Modulus for Multilayer Films in MEMS Applications
}

\author{
Xin-Ge Guo, Zai-Fa Zhou *, Chao Sun, Wei-Hua Li and Qing-An Huang \\ Key Laboratory of MEMS of the Ministry of Education, Southeast University, Nanjing 210096, China; \\ guoxinge93@outlook.com (X.-G.G.); sun_chaos@seu.edu.cn (C.S.); liwh@seu.edu.cn (W.-H.L.); \\ hqa@seu.edu.cn (Q.-A.H.) \\ * Correspondence: zfzhou@seu.edu.cn; Tel.: +86-025-8379-2632 (ext. 8817)
}

Received: 8 May 2017; Accepted: 20 June 2017; Published: 23 June 2017

\begin{abstract}
Based on the first resonance frequency measurement of multilayer beams, a simple extraction method has been developed to extract the Young's modulus of individual layers. To verify this method, the double-layer cantilever, as a typical example, is analyzed to simplify the situation and finite element modeling (FEM) is used in consideration of the buckling and unbuckling situation of cantilevers. The first resonance frequencies, which are obtained by ANSYS (15.0, ANSYS Inc., Pittsburgh, PA, USA) with a group of thirteen setting values of Young's modulus in the polysilicon layer are brought into the theoretical formulas to obtain a new group of Young's modulus in the polysilicon layer. The reliability and feasibility of the theoretical method are confirmed, according to the slight differences between the setting values and the results of the theoretical model. In the experiment, a series of polysilicon-metal double-layer cantilevers were fabricated. Digital holographic microscopy (DHM) (Lyncée Tech, Lausanne, Switzerland) is used to distinguish the buckled from the unbuckled. A scanning laser Doppler vibrometer (LDV) (Polytech GmbH, Berlin, Germany) system is used to measure the first resonance frequencies of them. After applying the measurement results into the theoretical modulus, the average values of Young's modulus in the polysilicon and gold layers are $151.78 \mathrm{GPa}$ and $75.72 \mathrm{GPa}$, respectively. The extracted parameters are all within the rational ranges, compared with the available results.
\end{abstract}

Keywords: resonance frequency; cantilever; FEM; thin film; Young's modulus

\section{Introduction}

Material mechanical parameters, such as Young's modulus, residual stress, and so on, not only have great effect on the functions of MEMS (Micro-Electro-Mechanical System) devices, but also have great influence on yield, service life, and the work reliability of MEMS devices. However, it is a challenging task to detect and measure the mechanical properties of thin films and membrane structures for MEMS applications [1-4]. In surface micromachining technology, with the same membrane structures, different processes will lead to different material properties. The different environment of a process, such as temperature and irradiation, will also cause different properties of the materials. Even from the same material, process, reactor, and environment when testing, there will also be differences among the material properties of thin films. Thus, the mechanical parameters need to be accurately measured in situ. As for the present measuring methods for mechanical parameters of thin film materials, most of them are suitable for single-layer thin film materials. For the micromachining technology of some materials, these methods for the single-layer thin film are no longer applicable. Therefore, an in situ extracting method of material properties $[5,6]$ for multilayer films is expected. Until now, various theoretical methods have been presented for extracting mechanical parameters of multilayer films, such as the resonance frequency method [7] and pull-in voltages [3,8]. 
In this paper, a novel approach was proposed to extract the Young's modulus of each layer with multilayer beams using resonance frequency measurement. Firstly, a model of the resonance frequency using multilayer beams with different widths was introduced. Then, taking the buckled and unbuckled situations of multilayer beams into consideration, a method was presented to extract the Young's modulus in each layer by designing test structures. The presented theoretical model was verified by FEM methods. Finally, some test structures, as a typical example, were fabricated and a scanning laser Doppler vibrometer (LDV) (Polytech GmbH, Berlin, Germany) system was used to measure their first resonance frequencies. The results turned out to be in good agreement with the rational values in the available literature. Without applying residual stress into the model and calculations, the test approaches and the calculation procedures became simple and easy.

\section{Theory}

\subsection{Theory Model of the Multilayer Beam}

The approach is to extract the Young's modulus of each layer based on multilayer beams, and the cross-section of an n-layer beam with different widths is shown in Figure 1. The length, width, and height direction of the beam are along the $x, y$, and $z$ axis, respectively. The length of the beam is $l$. The width, thickness, Young's modulus, Poisson's ratio, and density of the $i$ th layer are $w_{i}, h_{i}, E_{i}, v_{i}$, and $\rho_{i}$ respectively.

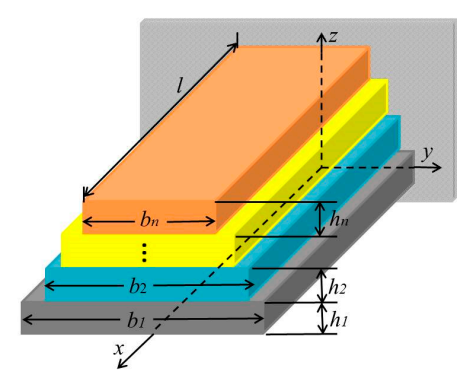

Figure 1. 3D view of an n-layer beam with different widths.

For composite multilayer beams studied in the paper, the widths are all five times longer than the thicknesses, and the stress-strain relation should be analyzed under the plain-strain condition. Thus, the Young's modulus $E_{i}$ should be substituted by the effective Young's modulus $\widetilde{E}_{i}$, as shown in Equation (1):

$$
\widetilde{E}_{i}=\left\{\begin{array}{cc}
E_{i} & , \quad w_{i}<5 h_{i} \\
\frac{E_{i}}{1-v_{i}{ }^{2}} & , \quad w_{i} \geq 5 h_{i}
\end{array}\right.
$$

As shown in Figure 1, the height of the bottom, $z_{0}$, is 0 and the height of the $i$ th layer is $z_{i}$. Thus, the expression of $z_{i}$ is shown in Equation (2):

$$
z_{i}=\sum_{j=1}^{i} h_{i}
$$

The distance of the neutral axis and the bottom of the beam is $z_{c}$, which is expressed as [9]:

$$
z_{c}=\frac{\sum_{i=1}^{n} \widetilde{E}_{i} w_{i}\left(z_{i}^{2}-z_{i-1}^{2}\right)}{2 \sum_{i=1}^{n} \widetilde{E}_{i} w_{i}\left(z_{i}-z_{i-1}\right)}
$$


The moment of inertia of the $i$ th layer, $I_{i}$, with respect to the neutral axis of the beam is expressed in Equation (4):

$$
I_{i}=\int_{i}\left(z-z_{\mathcal{c}}\right)^{2} d A_{i}=\frac{1}{3} w_{i}\left[\left(z_{i}-z_{\mathcal{c}}\right)^{3}-\left(z_{i-1}-z_{\mathcal{c}}\right)^{3}\right]
$$

Here $A_{i}$ is the cross-section area of the $i$ th layer. The bending stiffness $\overline{E I}$ and the linear density $\overline{\rho A}$ of the beam are shown below [10]:

$$
\begin{gathered}
\overline{E I}=\sum_{i=1}^{n} \widetilde{E}_{i} I_{i} \\
\overline{\rho A}=\sum_{i=1}^{n} \rho_{i} A_{i}
\end{gathered}
$$

\subsection{The Deduction of the Curvature Radius}

The paper takes multilayer cantilevers as the test structures. For a multilayer cantilever, if the compressive stress of the top layer is predominant, the cantilever will have an upward deflection. If the tensile stress of the top layer is predominant, the cantilever will have a downward deflection. Both the upward and downward deflection has the same effect on the modeling and results. However, if the downward deflection is too large to adhere to the substrate, cantilevers are difficult to, or cannot, be vibrated independently and the results from these structures are invalid and discarded. Therefore, the model proposed is suitable in small residual stress situations. Models, which can compensate the influence of the large residual stress in the thin films, are shown in [11-13].

The paper takes the upward deflection, for example, and assumes that the buckled multilayer cantilever has a uniform curvature radius, as shown in Figure 2. Figure 2 shows the relation between the curvature radius and the maximum deflection of the multilayer cantilever, as expressed by Equation (7):

$$
\left\{\begin{array}{c}
\theta=\quad l / R \\
\cos (\theta)=\left[R-\left(z_{1}-z_{c}\right)-z_{m}\right] /\left[R-\left(z_{1}-z_{c}\right)\right]
\end{array}\right.
$$

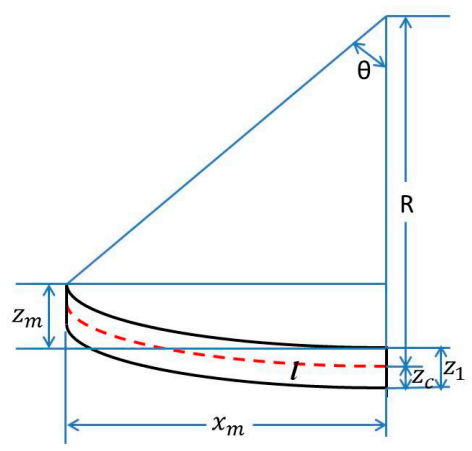

Figure 2. Schematic of a buckled multilayer beam.

In Figure 2, $R$ is the curvature radius of the multilayer cantilever, $z_{m}$ is the maximum deflection of cantilever, $l$ is the length of the unbuckled cantilever, $x_{m}$ is the length of buckled cantilever, $z_{1}$ is the thickness of the multilayer cantilever and $z_{c}$ is the height of the neutral axis. By solving Equation (7), values of the curvature radius can be obtained accurately. Generally, the maximum deflection of the beam is much smaller than the length of the beam, so $x_{m}$ can be approximated to $l$. Furthermore, $z_{1}-z_{\mathcal{c}}$ is also much smaller than the curvature radius of the cantilever, so after simplification and ignoring $z_{1}-z_{c}, z_{m}$ can be expressed by $z_{m}=R-R \cos \theta$. Using the half-angle formula $2 \sin ^{2} \frac{\theta}{2}=1-\cos \theta$ and 
the approximately simplification $\sin \frac{\theta}{2}=\frac{l}{2 R}$, a simplified equation, which is shown in Equation (8), can be obtained:

$$
R=l^{2} /\left(2 z_{m}\right)
$$

\subsection{The Theoretical Deduction for Material Parameters}

If the longitudinal gradient residual stress is neglected, the single-layer cantilever will remain unbuckled after release. The strain resulting from the residual stress tends to have less effect on the vibration. Therefore, it is considered that the resonance frequency has nothing to do with the residual stress when the single-layer cantilever remains unbuckled after release [14]. However, due to the unmatched thermal stress, or other reasons, in most cases the multilayer cantilever will be buckled after release. The deflection has an effect on the resonance frequency of the cantilever after release $[15,16]$.

For unbuckled multilayer cantilevers after release, the approximate analytic formula of its first resonant frequency is shown in Equation (9):

$$
f_{1 u c f}=\frac{1.875^{2}}{2 \pi l^{2}} \sqrt{\frac{\overline{E I}}{\overline{\rho A}}}
$$

here subscript 1 represents the first resonant frequency, $u$ represents that the cantilever is unbuckled, and $c f$ represents that the boundary condition is cantilever.

When the cantilever is buckled, the influence of deflection to resonance frequency cannot be ignored. Assuming that $R$ is the curvature radius of cantilever after release, a function describing its first resonant frequency is shown in Equation (10) [15-17]:

$$
\begin{gathered}
\frac{1}{\sqrt{\chi_{n}^{2}-1}} \sin \left(\sqrt{\chi_{n}+1} \frac{l}{R}\right) \sinh \left(\sqrt{\chi_{n}-1} \frac{l}{R}\right)+ \\
\cos \left(\sqrt{\chi_{n}+1} \frac{l}{R}\right) \cosh \left(\sqrt{\chi_{n}-1} \frac{l}{R}\right)+1=0,
\end{gathered}
$$

where:

$$
\chi_{n}^{2}=\overline{\rho A} R^{4}\left(2 \pi f_{n}\right)^{2} / \overline{E I} .
$$

Equation (10) is a transcendental equation. Except rare cases (such as the simple trigonometric equation), this kind of equation can only be solved approximately using numerical methods. There are many approximate methods to solve the transcendental equation. Common methods are Newton's method, dichotomy, Mueller's method, and so on $[18,19]$. There are numerous sets of solutions $\chi_{n}$ for Equation (13). Each $\chi_{n}$ has the corresponding $i$ th resonance frequency. However, to ensure that the first resonance frequency $f_{1}$ is obtained, the solution of Equation (10) should be the smallest one, $\chi_{1}$.

If the curvature radius of the cantilever tends to infinity, take the limit of Equation (10) and the result is the same as Equation (9), which means when the curvature radius of the cantilever tends to infinity, the vibrations of both the buckled and unbuckled multilayer cantilever have little difference. Thus, in this situation, Equation (9) can describe its resonance frequency approximately.

\subsection{The Extraction of Material Parameters}

Under buckled or unbuckled situations of $n$-layer cantilevers, it only needs $n$ types of cantilevers to obtain the Young's modulus of each layer. The widths of the $j$ th cantilever are $w_{j 1}, w_{j 2}, \ldots, w_{j n}$ and the widths of the $k$ th cantilever are $w_{k 1}, w_{k 2}, \ldots, w_{k n}$. If they have the same length, the vector $\left(w_{j 1}\right.$, $\left.w_{j 2}, \ldots, w_{j n}\right)$ and the vector $\left(w_{k 1}, w_{k 2}, \ldots, w_{k n}\right)$ should be linearly independent [20]. DHM (Lyncée Tech, Lausanne, Switzerland) was used to distinguish the buckled from the unbuckled and determined 
using Equation (9) or Equation (11). By applying the smallest value $\chi_{1}$ into Equation (11), the changed form of Equation (11) is shown in Equation (12):

$$
f_{1 b c f}=\frac{1}{2 \pi} \cdot\left(\frac{\chi_{1}^{2} \cdot \overline{E I}}{\overline{\rho A} \cdot R^{4}}\right)^{1 / 2}
$$

Here, $b$ represents that the cantilever is buckled. Assuming that the first resonance frequencies measured are $f_{1}, f_{2}, \ldots, f_{n}$, a set of equations is deduced from the theoretical formulas, as shown in Equation (13):

$$
\left\{\begin{array}{c}
f_{1 x c f, 1}\left(\widetilde{E}_{1}, \widetilde{E}_{2}, \ldots, \widetilde{E}_{n}\right)-f_{1}=0 \\
f_{1 x c f, 2}\left(\widetilde{E}_{1}, \widetilde{E}_{2}, \ldots, \widetilde{E}_{n}\right)-f_{2}=0 \\
f_{1 x c f, n}\left(\widetilde{E}_{1}, \widetilde{E}_{2}, \ldots, \widetilde{E}_{n}\right)-f_{n}=0
\end{array}\right.
$$

where:

$$
x=\left\{\begin{array}{l}
b, \text { when the cantilaver is buckled. } \\
u, \text { when the cantilaver is unbuckled. }
\end{array}\right.
$$

Since each equation in Equation (13) is the linear equation, it can be shown in matrix form, which is shown in Equation (14):

$$
A\left[\begin{array}{c}
\widetilde{E}_{1} \\
\widetilde{E}_{2} \\
\cdots \\
\widetilde{E}_{n}
\end{array}\right]=\left[\begin{array}{l}
f_{1}^{2} \\
f_{2}^{2} \\
\cdots \\
f_{n}^{2}
\end{array}\right]
$$

where:

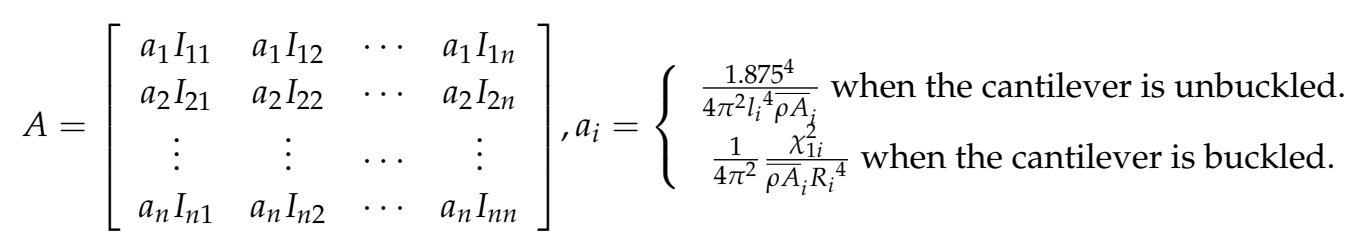

$I_{i j}$ represents the moment of inertia of $j$ th layer of the $i$ th cantilever. $l_{i}, \overline{\rho A}_{i}, \chi_{1 i}^{2}$, and $R_{i}$ represent the length, the linear density, the smallest value $\chi_{1}$, and the curvature radius of the $i$ th cantilever, respectively. Since vectors which consist of widths of all of the cantilevers are linearly independent, the matrix A is invertible. Therefore, Equation (13) is solvable.

Effective Young's modulus $\widetilde{E}_{i}$ will be obtained from Equation (13) rather than Young's modulus $E_{i}$ because the Poisson's ratio $v_{i}$ in each layer is unknown. To make results more intuitive, the paper sets exact values to the Poisson's ratio in each layer. The way to obtain Poisson' ratio $v_{i}$ is shown in [21-23]. The Poisson's ratio of each layer in a certain rational range has little effect on the results of the Young's modulus. For example, the rational range of the Poisson's ratio in the polysilicon layer is $0.2 \sim 0.25$ and the paper assumes 0.22 as the Poisson's ratio in the polysilicon layer. It is obvious that results obtained from this will have little differences among the results obtained from the Poisson's ratio in the polysilicon layer is 0.2 or other values in this rational range.

Solving Equation (13) will give many sets of results, but only one of them is required. Thus, it is necessary to select only one rational result from them. For example, the Young's modulus of polysilicon has been reported in the range of $120 \mathrm{GPa} 201 \mathrm{GPa}$ [20]. Thus, only one result in this range is correct.

\section{Finite Element Modeling}

The double-layer cantilever adopted in this paper can simplify the situation. To verify the validity of the theoretical model, a set of double-layer cantilevers was analyzed by the FEM method. Since 
ANSYS (15.0, ANSYS Inc., Pittsburgh, PA, USA) cannot express the residual stress after release, heating the structure in advance simulated the buckled cantilever. From bottom to top, the first layer of the cantilevers was designed to be a polysilicon material, and the second layer was designed to be metal material: gold. Assuming that the parameters of the metal layer are fixed, the only changes to the Young's modulus are in the polysilicon layer.

An available range of Young's modulus of polysilicon is from $120 \mathrm{GPa}$ to $180 \mathrm{GPa}$. This paper takes thirteen values between it with the interval of $5 \mathrm{GPa}$ and obtains thirteen values of the first resonance frequency and the maximum deflection of the cantilevers, respectively, for theoretical deduction. Dimensions and parameters of the cantilevers (except the Young's modulus of the polysilicon layer) are shown in Table 1.

Table 1. Dimensions and parameters of the cantilevers.

\begin{tabular}{ccc}
\hline Dimension/Parameter & Polysilicon Layer & Metal Layer \\
\hline Young's modulus $E_{\boldsymbol{i}}(\mathrm{GPa})$ & - & 57 \\
Residual stress $\sigma_{\boldsymbol{i}} \mathbf{( M P a )}$ & 10 & -20 \\
Poisson ratio $\boldsymbol{v}_{\boldsymbol{i}}$ & 0.22 & 0.35 \\
Density $\boldsymbol{\rho}_{\boldsymbol{i}}\left(\mathbf{k g} / \mathbf{m}^{\mathbf{3}}\right)$ & 2330 & 19,300 \\
Length $l(\mu \mathrm{m})$ & 200 & 200 \\
Width $w_{\boldsymbol{i}}(\mu \mathrm{m})$ & 30 & 30 \\
\hline
\end{tabular}

Taking the Young's modulus in the polysilicon layer as $120 \mathrm{GPa}$ as an example, pictures of a polysilicon-metal double-layer cantilever structure and pictures of the deflection values of the cantilever (shown in Figure 3) can be obtained by using ANSYS (15.0, ANSYS Inc., Pittsburgh, PA, USA) in both unbuckled and buckled situations.

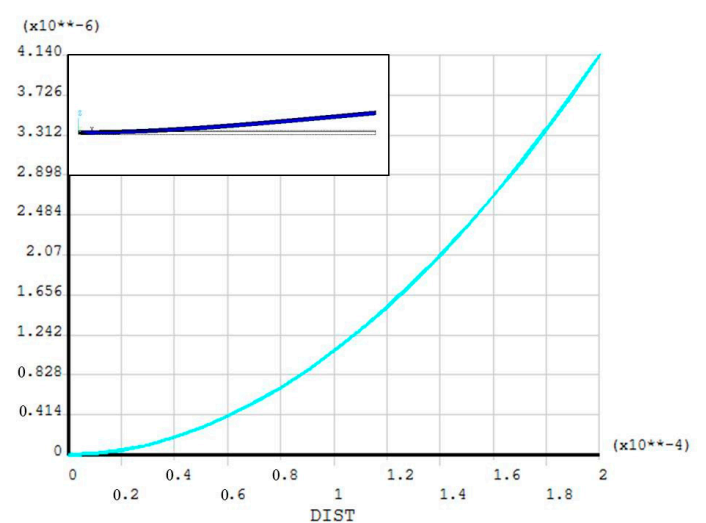

(a)

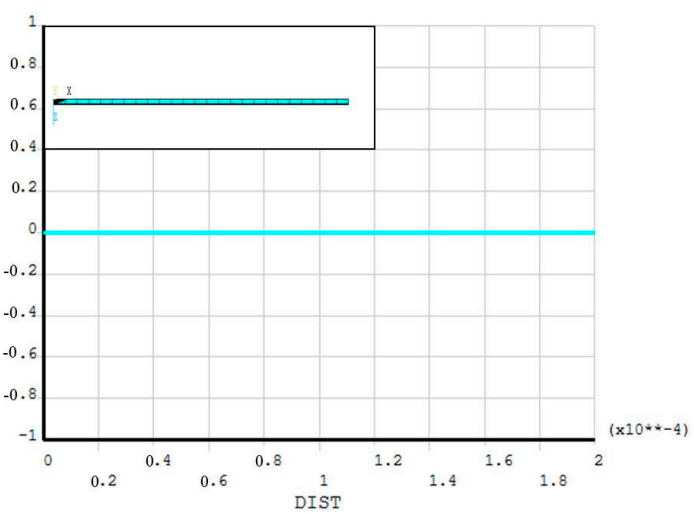

(b)

Figure 3. Pictures of the structure and deflection values of the cantilever. (a) The cantilever is buckled. (b) The cantilever is unbuckled.

\section{Simulation Results}

When the difference between the curvature radius of the cantilever and the length of the cantilever is not obvious, the cantilever has a large deflection. In this case, through its curvature radius and the first resonance frequency, which is from ANSYS (15.0, ANSYS Inc., Pittsburgh, PA, USA), theoretical values of the Young's modulus in the polysilicon layer can be deduced accurately by solving Equations (10) and (11).

When the cantilever has a small deflection, the deflection can be ignored approximately. In this case, the theoretical values of the Young's modulus in the polysilicon layer can be obtained by using Equation (9) through its first resonance frequency of the cantilever. 
In both buckled and unbuckled situations of the cantilevers, the first resonance frequency is obtained by ANSYS (15.0, ANSYS Inc., Pittsburgh, PA, USA), the maximum deflection of the cantilevers, the curvature radius deduced, the theoretical values of the Young's modulus in the polysilicon layer and the comparison between setting values of the Young's modulus in the polysilicon layer, as well as ones in the theoretical model, are shown in Tables 2 and 3.

Table 2. Results when the cantilever is buckled.

\begin{tabular}{|c|c|c|c|c|c|}
\hline $\begin{array}{l}\text { Setting Values } \\
\text { of Young's } \\
\text { Modulus in } \\
\text { Polysilicon } \\
\text { Layer E (GPa) }\end{array}$ & $\begin{array}{c}\text { The First } \\
\text { Resonance } \\
\text { Frequency } \\
\text { from ANSYS } \\
(\mathrm{Hz})\end{array}$ & $\begin{array}{l}\text { The Maximum } \\
\text { Deflection of } \\
\text { Cantilever } \\
\text { from ANSYS } \\
(\mu \mathrm{m})\end{array}$ & $\begin{array}{c}\text { The Curvature } \\
\text { Radius ( } \mu \mathrm{m})\end{array}$ & $\begin{array}{c}\text { Theoretical Values } \\
\text { of Young's } \\
\text { Modulus in the } \\
\text { Polysilicon Layer } \\
E^{\prime} \text { (GPa) }\end{array}$ & $\begin{array}{c}\text { Error (\%) } \\
\left|\left(\boldsymbol{E}-\boldsymbol{E}^{\prime}\right) / \boldsymbol{E}\right|\end{array}$ \\
\hline 120 & 30,076 & 4.1403 & 4830.57 & 110.591 & 7.841 \\
\hline 125 & 30,466 & 4.0334 & 4958.60 & 115.353 & 7.718 \\
\hline 130 & 30,848 & 3.9237 & 5085.56 & 120.114 & 7.605 \\
\hline 135 & 31,223 & 3.8376 & 5211.59 & 124.877 & 7.499 \\
\hline 140 & 31,593 & 3.7476 & 5336.75 & 129.664 & 7.383 \\
\hline 145 & 31,956 & 3.6622 & 5461.20 & 134.443 & 7.281 \\
\hline 150 & 32,313 & 3.5810 & 5585.03 & 139.222 & 7.185 \\
\hline 155 & 32,665 & 3.5038 & 5708.09 & 144.008 & 7.092 \\
\hline 160 & 33,013 & 3.4301 & 5830.73 & 148.813 & 6.992 \\
\hline 165 & 33,355 & 3.3598 & 5952.74 & 153.604 & 6.907 \\
\hline 170 & 33,693 & 3.2926 & 6074.23 & 158.406 & 6.820 \\
\hline 175 & 34,027 & 3.2283 & 6195.21 & 163.216 & 6.734 \\
\hline 180 & 34,356 & 3.1667 & 6315.72 & 168.016 & 6.658 \\
\hline
\end{tabular}

Table 3. Results when the cantilever is unbuckled.

\begin{tabular}{cccc}
\hline $\begin{array}{c}\text { Setting Values of Young's } \\
\text { Modulus in Polysilicon } \\
\text { Layer } \boldsymbol{E} \text { (GPa) }\end{array}$ & $\begin{array}{c}\text { The First Resonance } \\
\text { Frequency from ANSYS } \\
\mathbf{( H z )}\end{array}$ & $\begin{array}{c}\text { Theoretical Values of } \\
\text { Young's Modulus in the } \\
\text { Polysilicon Layer } \boldsymbol{E}^{\prime \prime}(\mathbf{G P a})\end{array}$ & $\begin{array}{c}\text { Error (\%) } \\
\mid\left(\boldsymbol{E}-\boldsymbol{E}^{\prime \prime}\right) / \boldsymbol{E}^{\prime}\end{array}$ \\
\hline 120 & 30,074 & 110.650 & 7.792 \\
125 & 30,464 & 115.413 & 7.670 \\
130 & 30,846 & 120.173 & 7.560 \\
135 & 31,221 & 124.937 & 7.454 \\
140 & 31,590 & 129.711 & 7.349 \\
145 & 31,954 & 134.503 & 7.239 \\
150 & 32,311 & 139.282 & 7.145 \\
160 & 32,663 & 144.069 & 7.052 \\
165 & 33,011 & 148.874 & 6.954 \\
170 & 33,353 & 153.665 & 6.870 \\
180 & 33,691 & 158.468 & 6.784 \\
\end{tabular}

From Tables 2 and 3, the differences between the setting values of the Young's modulus in the polysilicon layer and the values in the theoretical model are both not obvious. As the Young's modulus in the polysilicon layer increases, the curvature radius of the cantilever increases. For relatively large curvature radii, errors from the calculations have less of an effect on its veracity. Thus final errors decrease as the Young's modulus in the polysilicon layer increases. In addition, comparing the buckled situation with the unbuckled situation, slight differences between the theoretical values of the Young's modulus in the polysilicon layer and errors in the unbuckled situation are all less than the ones in the buckled situation. This means that the cantilever does not have much deflection and using Equation (9), therefore, is more accurate. The reliability and feasibility of the theoretical model is demonstrated by slight errors which are all less than $8 \%$.

Some of the reasons why setting values and theoretical values have differences are as follows. Firstly, conditions in the theoretical model and the actual ones are not exactly the same. Secondly, when 
simulating, there might exist some uncontrollable factors which lead to the simulation environment being different from the ideal environment. Thirdly, the deduction of the curvature radius of the cantilever will bring in inevitable errors.

\section{Experiments and Discussion}

Test structures, which are double-layer cantilevers for simplification, were fabricated using the MEMSCAP PolyMUMPS (MEMSCAP Inc., Durham, NC, USA) process based on surface micromachining technology. Before measuring the first resonance frequency with an LDV (Polytech $\mathrm{GmbH}$, Berlin, Germany) system with a hardware modulus of MSV-400-M2, DHM (Lyncée Tech, Lausanne, Switzerland) (R2200) was used to determine whether cantilevers had deflection and to obtain the values of the curvature radius. Principles of LDV (Polytech GmbH, Berlin, Germany) and DHM (Lyncée Tech, Lausanne, Switzerland) are provided in the Supplementary Materials.

For the double-layer cantilever, there are two unknown values of the Young's modulus to obtain. Thus, two double-layer cantilevers with the same length but different widths should be grouped for calculation. The double-layer cantilevers were designed in two different lengths, and with the same length, widths of two double-layer cantilevers should be linearly independent. Cantilever 4, as an example, was shown in Figure 4 in scanning electron microscopy (SEM) photographs. The dimension, the deformation by DHM (Lyncée Tech, Lausanne, Switzerland), and the first resonance frequency measured of the cantilevers are shown in Table 4. To avoid the situation that the bottom layer of the cantilever adheres to the substrate, test structures with suitable dimensions should be designed. Simulated by ANSYS (15.0, ANSYS Inc., Pittsburgh, PA, USA), cantilever 1, whose residual stress is $-20 \mathrm{MPa}$ in the polysilicon layer and $20 \mathrm{MPa}$ in the metal layer, have a downward deflection of $-1.4304 \mu \mathrm{m}$. Residual stress of $20 \mathrm{MPa}$ is enough to have a large deflection. Thus, compared to the thickness of the sacrificial layer of $2 \mu \mathrm{m}$, cantilevers, whose dimensions are little different from that of cantilever 1, may guarantee a relatively small possibility of substrate adhesion. However, it should be noted that the suitable dimension mentioned above is applicable to the MEMSCAP PolyMUMPS (MEMSCAP Inc., Durham, NC, USA) process and the dimensions of the test structures should be adjusted according to the specific process. In fact, a short time on release, which results when the multilayer cantilevers are relatively narrow, and the relatively large stiffness of multilayer cantilevers, leads to little possibility of adhesion.

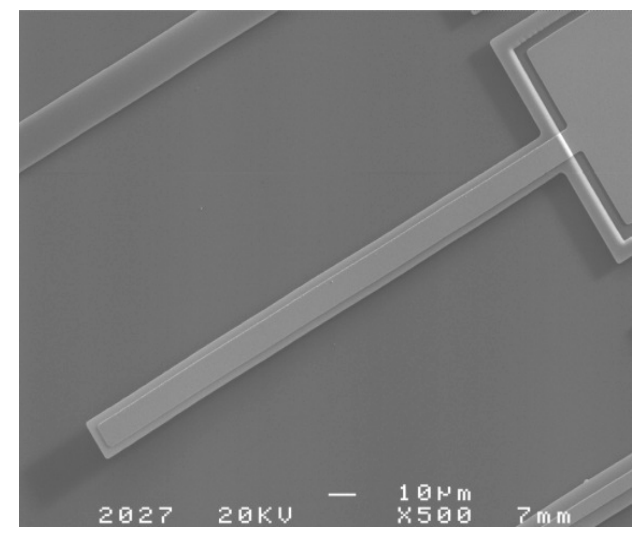

Figure 4. A SEM picture of cantilever 4.

Assuming that the cantilever has a uniform curvature radius $R$, curvature radii are obtained by numerical fitting from the deflection curves. The obtained curvature radii are shown in Table 5. 
Table 4. Parameters of the cantilevers.

\begin{tabular}{|c|c|c|c|c|c|c|c|}
\hline Cantilever $i$ & $\begin{array}{l}\text { Length } \\
l_{i}(\mu \mathrm{m})\end{array}$ & $\begin{array}{l}\text { Width of the } \\
\text { Polysilicon } \\
\text { Layer } w_{1 i} \\
\quad(\mu \mathrm{m})\end{array}$ & $\begin{array}{l}\text { Width of } \\
\text { Metal } \\
\text { Layer } w_{2 i} \\
\quad(\mu \mathrm{m})\end{array}$ & $\begin{array}{c}\text { Thickness of } \\
\text { the } \\
\text { Polysilicon } \\
\text { Layer } h_{1}(\mu \mathrm{m})\end{array}$ & $\begin{array}{l}\text { Thickness of } \\
\text { the Metal } \\
\text { Layer } h_{2}(\mu \mathrm{m})\end{array}$ & $\begin{array}{c}\text { Initially } \\
\text { Buckled } \\
\text { or } \\
\text { Unbuckled }\end{array}$ & $\begin{array}{c}\text { The First } \\
\text { Resonance } \\
\text { Frequency } \\
f_{i}(\mathbf{k H z})\end{array}$ \\
\hline Cantilever 1 & 150 & 15 & 5 & 1.5 & 0.5 & buckled & 74.38 \\
\hline Cantilever 2 & 150 & 15 & 9 & 1.5 & 0.5 & buckled & 68.28 \\
\hline Cantilever 3 & 200 & 15 & 5 & 1.5 & 0.5 & buckled & 41.72 \\
\hline Cantilever 4 & 200 & 15 & 9 & 1.5 & 0.5 & buckled & 38.75 \\
\hline
\end{tabular}

Table 5. Results of numerical fitting for cantilevers.

\begin{tabular}{lcccccc}
\hline Cantilever $\boldsymbol{i}$ & $\begin{array}{c}\text { Curvature } \\
\text { Radius } \boldsymbol{R} \\
(\boldsymbol{\mu m})\end{array}$ & $\begin{array}{c}\text { Standard } \\
\text { Deviation } \\
\sigma_{\boldsymbol{R}}(\boldsymbol{\mu m})\end{array}$ & $\begin{array}{c}\text { Abscissa of } \\
\text { the Anchor } \\
\text { End } \boldsymbol{x}_{\mathbf{0}}(\boldsymbol{\mu m})\end{array}$ & $\begin{array}{c}\text { Standard } \\
\text { Deviation } \\
\sigma_{\boldsymbol{x}}(\boldsymbol{\mu m})\end{array}$ & $\begin{array}{c}\text { Ordinate of } \\
\text { the Anchor } \\
\text { End } \boldsymbol{z}_{\mathbf{0}}(\boldsymbol{\mu m})\end{array}$ & $\begin{array}{c}\text { Standard } \\
\text { Deviation } \\
\sigma_{\boldsymbol{z}}(\boldsymbol{\mu m})\end{array}$ \\
\hline Cantilever 1 & $12,997.32$ & 24.60 & 2.98 & $1.40 \times 10^{-1}$ & $5.06 \times 10^{-3}$ & $3.34 \times 10^{-4}$ \\
Cantilever 2 & 9690.63 & 14.89 & -1.37 & $1.20 \times 10^{-1}$ & $1.73 \times 10^{-3}$ & $4.09 \times 10^{-4}$ \\
Cantilever 3 & $13,011.4$ & 12.10 & 2.27 & $0.93 \times 10^{-1}$ & $3.31 \times 10^{-3}$ & $3.03 \times 10^{-4}$ \\
Cantilever 4 & 9587.3 & 7.33 & -0.94 & $0.79 \times 10^{-1}$ & $1.86 \times 10^{-3}$ & $3.61 \times 10^{-4}$ \\
\hline
\end{tabular}

Assuming that the Poisson' ratio and density of the polysilicon layer are 0.22 and $2330 \mathrm{~kg} / \mathrm{m}^{3}$, respectively, and the Poisson' ratio and density of the metal are 0.44 and $19,300 \mathrm{~kg} / \mathrm{m}^{3}$, respectively, the Young's modulus of each layer can be calculated by the first resonance frequencies of the test structures. Here is an example to use cantilever 1 and cantilever 2 to extract the Young's modulus of each layer. After applying the material parameters and the first resonance frequencies in Equations (10) and (11), a system of two-element equations can be obtained, as shown in Equation (15):

$$
\left\{\begin{array}{l}
f_{1 b c f, 1}\left(\widetilde{E}_{1}, \widetilde{E}_{2}\right)-f_{1}=0 \\
f_{1 b c f, 2}\left(\widetilde{E}_{1}, \widetilde{E}_{2}\right)-f_{2}=0
\end{array}\right.
$$

Solve Equation (15) and find the only answer which meets the practical range of the material parameters. The reasonable answer is that the values of the Young's modulus in the polysilicon layer and in the metal layer are $156.77 \mathrm{GPa}$ and $68.54 \mathrm{GPa}$, respectively. Similarly, the Young's modulus of each layer can also be extracted by cantilever 3 and cantilever 4 . The results are shown in Table 6 . From Table 6 , it is obvious that the results agree with the practical range of material parameters and the values reported in [24].

Table 6. Results in different sets of cantilevers.

\begin{tabular}{ccc}
\hline Cantilever $\boldsymbol{i}$ & $\begin{array}{c}\text { Young's Modulus in the } \\
\text { Polysilicon Layer } \boldsymbol{E}_{\mathbf{1}} \mathbf{( G P a )}\end{array}$ & $\begin{array}{c}\text { Young's Modulus in the Metal } \\
\text { Layer } \boldsymbol{E}_{\mathbf{2}} \mathbf{( G P a )}\end{array}$ \\
\hline Cantilever 1 and 2 & 156.77 & 68.54 \\
Cantilever 3 and 4 & 146.78 & 82.89 \\
Average value & 151.78 & 75.72 \\
Reference range reported in [24] & $120 \sim 201$ & 78 \\
\hline
\end{tabular}

There are some issues that affect the accuracy of the results. Firstly, in practical processes, photoetching, masking, and self-aligned processes can lead to dimension deviation between designed structures and processed ones. Generally, in surface micromachining technology, dimension deviations in a plane are no more than $0.5 \mu \mathrm{m}$ and dimension deviations in thickness are no more than $0.02 \mu \mathrm{m}$. It will have an inevitable influence on minimized devices. Secondly, squeezed damping in air may lead to the inaccuracy of the first resonance frequency of the measured cantilevers $[25,26]$. Thus, the amplification factor for the frequency response functions at points of the resonance frequencies are needed to be greater than 20 [20]. 


\section{Conclusions}

In this paper, an approach of extracting the Young's modulus of each layer for multilayer films was developed. Based on the first resonance frequency, a theoretical model was proposed for the multilayer beam. The multilayer cantilevers were adopted as the test structures, and buckled or unbuckled situations of the cantilevers were both considered. Its reliability and feasibility were confirmed theoretically by the FEM method with less than $8 \%$ error. In experiments, double-layer cantilevers have been fabricated for simplification. The Young's modulus of each layer can be obtained by using two double-layer cantilevers with the same length, but different widths. The experimental results prove the accuracy of the presented approaches, and this study is suitable to extract the Young's modulus of each layer for multilayer films.

Supplementary Materials: The following are available online at http:/ /www.mdpi.com/2072-666X/8/7/201/s1, Figure S1: (a) A schematic of the laser Doppler vibrometer (LDV); (b) A picture of LDV system, Figure S2: (a) A schematic of the digital holographic microscopy (DHM); (b) A picture of DHM.

Acknowledgments: Support by the National High Technology Program of P. R. China under grant No. 2015AA042604 is gratefully acknowledged.

Author Contributions: Xin-Ge Guo and Zai-Fa Zhou developed the model and designed the experiments; Xin-Ge Guo performed the experiments; Chao Sun contributed materials/analysis tools; and Qing-An Huang and Wei-Hua Li proposed the idea for the model and analyzed the data. Xin-Ge Guo and Zai-Fa Zhou edited this manuscript.

Conflicts of Interest: The authors declare no conflict of interest. The founding sponsors had no role in the design of the study; in the collection, analyses, or interpretation of data; in the writing of the manuscript, and in the decision to publish the results.

\section{References}

1. Lee, C.S.; Lee, J.H.; Choi, C.A.; No, K.; Wee, D.M. Effects of phosphorus on stress of multi-stacked polysilicon film and single crystalline silicon. J. Micromech. Microeng. 1999, 9, 252-263. [CrossRef]

2. Yoshikawa, E.; Tsugai, M.; Horikawa, M.; Otani, H.; Hamada, S. Influence of RTA parameters on residual stress and stress gradient of multilayered LPCVD polysilicon film. In Proceedings of the Fifteenth IEEE International Conference on Micro Electro Mechanical Systems, Las Vegas, NV, USA, 24 January 2002; pp. $451-454$.

3. Rong, H.; Huang, Q.-A.; Nie, M.; Li, W.-H. An analytical model for pull-in voltage of clamped-clamped multilayer beams. Sens. Actuators A Phys. 2004, 116, 15-21. [CrossRef]

4. Ilic, B.; Krylov, S.; Craighead, H. Determination of density and young's modulus of atomic layer deposited thin films by resonant frequency measurements of optically excited nanocantilevers. In Proceedings of the IEEE 22nd International Conference on Micro Electro Mechanical Systems (MEMS 2009), Sorrento, Italy, 25-29 January 2009; pp. 650-653.

5. Senturia, S.D. Cad challenges for microsensors, microactuators, and microsystems. Proc. IEEE 1998, 86, 1611-1626. [CrossRef]

6. Nie, M.; Huang, Q.-A.; Li, W.-H. Measurement of residual stress in multilayered thin films by a full-field optical method. Sens. Actuators A Phys. 2006, 126, 93-97. [CrossRef]

7. Nagel, D.J.; Zaghloul, M.E. Mems: Micro technology, mega impact. IEEE Circuits Devices Mag. 2001, 17, 14-25. [CrossRef]

8. Nie, M.; Huang, Q.-A.; Li, W.-H. Pull-in characterization of doubly-clamped composite beams. Sens. Actuators A Phys. 2009, 151, 118-126. [CrossRef]

9. Gere, J.M.; Goodno, B.J. Mechanics of materials (cengage learning, toronto, 2009). Chap 2009, 5, 356.

10. Marshall, J.C.; Herman, D.L.; Vernier, P.T.; DeVoe, D.L.; Gaitan, M. Young's modulus measurements in standard ic cmos processes using mems test structures. IEEE Electr. Device Lett. 2007, 28, 960-963. [CrossRef]

11. Phan, H.-P.; Nguyen, T.-K.; Dinh, T.; Ginosuke, I.; Kermany, A.R.; Qamar, A.; Han, J.; Namazu, T.; Ryutaro, M.; Dao, D.V. Ultra-high strain in epitaxial silicon carbide nanostructures utilizing residual stress amplification. arXiv 2017. Available online: https:/ / arxiv.org/abs/1701.02791 (accessed on 21 June 2017). 
12. Changizi, A.; Stiharu, I.; Olbrechts, B.; Raskin, J.-P. Extraction method for the residual stress in multilayer microplates under large deflection based on static deflection analysis. J. Microelectromech. Syst. 2015, 24, 1150-1163. [CrossRef]

13. Kozeki, T.; Phan, H.-P.; Dao, D.V.; Inoue, S.; Namazu, T. Influence of gallium ion beam acceleration voltage on the bend angle of amorphous silicon cantilevers. Jpn. J. Appl. Phys. 2016, 55, 06GL02. [CrossRef]

14. Osterberg, P.M.; Senturia, S.D. M-test: A test chip for mems material property measurement using electrostatically actuated test structures. J. Microelectromech. Syst. 1997, 6, 107-118. [CrossRef]

15. Shen, F.; Lu, P.; O'Shea, S.; Lee, K.; Ng, T. Thermal effects on coated resonant microcantilevers. Sens. Actuators A Phys. 2001, 95, 17-23. [CrossRef]

16. Henrych, J. The Dynamics of Arches and Frames; Elsevier Science Ltd.: Amsterdam, The Netherlands, 1981; Volume 2, pp. 33-108.

17. Sun, C. Study on In-Situ Extracting Method for Mechanical Properties of CMOS MEMS Multilayered Films. Ph.D. Thesis, Southeast University, Nanjing, China, November 2014. (In Chinese)

18. Traub, J.F. Iterative Methods for the Solution of Equations; American Mathematical Soc.: Providence, RI, USA, 1982.

19. Ostrowski, A.N. Solutions of Equations in Euclidean and Banach Spaces; Academic Press: Cambridge, MA, USA, 1973.

20. Sun, C.; Zhou, Z.-F.; Li, W.-H.; Huang, Q.-A. A simple method for extracting material parameters of multilayered mems structures using resonance frequency measurements. J. Micromech. Microeng. 2014, 24, 075014. [CrossRef]

21. Sun, J.-Y.; Hu, J.-L.; Zheng, Z.-L.; He, X.-T.; Geng, H.-H. A practical method for simultaneous determination of poisson's ratio and young's modulus of elasticity of thin films. J. Mech. Sci. Technol. 2011, 25, 3165-3171. [CrossRef]

22. Namazu, T.; Fujii, T.; Takahashi, M.; Tanaka, M.; Inoue, S. A simple experimental technique for measuring the poisson's ratio of microstructures. J. Microelectromech. Syst. 2013, 22, 625-636. [CrossRef]

23. Li, L.; Gomes, J.; Brown, G.; Uttamchandani, D.; Pan, W.; Weiland, D.; Begbie, M.; Lowrie, C.; Desmulliez, M.P. Simultaneous determination of the young's modulus and poisson's ratio in micro/nano materials. J. Micromech. Microeng. 2009, 19, 125027. [CrossRef]

24. Material: Polysilicon, Film. Available online: https://www.memsnet.org/material/polysiliconfilm/ (accessed on 28 April 2017).

25. Song, J.; Huang, Q.-A.; Li, M.; Tang, J.-Y. Effect of die-bonding process on mems device performance: System-level modeling and experimental verification. J. Microelectromech. Syst. 2009, 18, 274-286. [CrossRef]

26. Kobrinsky, M.J.; Deutsch, E.R.; Senturia, S.D. Effect of support compliance and residual stress on the shape of doubly supported surface-micromachined beams. J. Microelectromech. Syst. 2000, 9, 361-369. [CrossRef] 